\title{
What Do Patients Value in the Hospital Meal Experience?
}

Heather J Hartwell PhD

Paula A Shepherd PhD

John S.A. Edwards PhD

Nick Johns PhD*

Food Service and Applied Nutrition Research Group, Bournemouth University, Talbot Campus, Poole, Dorset, BH12 5BB

*Corresponding Author

njohns68@tiscali.co.uk

Telephone: + $44(0) 1603305815$

Authorship

This paper contains data collected as part of the PhD of Paula Shepherd as supervised by Dr Hartwell and Professor Edwards, data analysis was conducted by Dr Johns. Authorship was based on substantive contributions to each of the following: conception and design of the study; generation, collection, assembly, analysis and/or interpretation of data; drafting and revision of the manuscript and approval of the final version.

Word count: 4,654

Number of figures and tables: Figures 2; Tables 3

Key words: Hospitals; Food Service; Meal experience; Patient satisfaction

Abstract

A number of previous studies have reported on the aspects of hospital food service that patients value, but usually as a secondary finding, and not generally based upon patient-centred approaches. 
This study employed a questionnaire produced ab initio from interviews with patients and hospital staff, the data from which were subjected to factor and cluster analysis, in order to identify and prioritise the factors that contribute to the meal experience empirically. The most important factors, food and service were as identified by other authors. In decreasing order of importance were social, personal and situational factors. The results confirm that improving the quality of the food and the efficiency with which it reaches the patients remain the most important objectives of hospital food service. 
Inadequate nutrition of patients is common in all types of hospitals, all types of wards, and among all diagnostic categories and ages (BAPEN 2007). It has been shown to increase the incidence of postoperative complications and the need for drugs and other interventions (Feldblum et al. 2009) and as a result it may lengthen the typical stay in hospital by $50 \%$ (European Nutrition for Health Alliance 2008). Causes of inadequate nutrition in hospitals include the quality and appearance of the food and the eating environment, factors that contribute to the whole meal experience. Parallel with concerns about malnutrition, consumer expectations of hospitals have been increasing, so that the provision of food and the meal experience are becoming increasingly important within the range of medical and support services offered by hospitals (Andersson et al. 2013; Russell et al. 2011; Spencer \& Walshe, 2009). One part of managing and maintaining hospital food service standards involves assessing patients' satisfaction, and various studies have been conducted in different countries (Bélanger \& Dubé 1996; Fallon et al. 2007; Hwang et al. 2003; Sahin et al. 2006; Jessri et al. 2011). These vary in scope and have involved both quantitative and qualitative approaches, but the stated aim has always been to gain insight on patients' service experience.

Patients' satisfaction with food service in hospitals is commonly assessed using questionnaires. This approach is exemplified by a Canadian group led by Dubé and Bélanger, who in the late 1990s developed a questionnaire for assessing acute patients' satisfaction with hospital meals (Bélanger \& Dubé, 1996; Dubé et al. 1994). Significantly, their study considered emotional aspects of the meal experience, but the questionnaire itself was based upon previous literature, rather than on a related, qualitative study. Statistical analysis of the survey results of Dubé et al. (1994) identified the seven factors shown in Table 1. The authors note that food quality was the best predictor of patient satisfaction, followed by customization and attitude of the staff who deliver menus. An Australian group led by Capra (Wright et al. 2003; Capra et al., 2005) developed an Acute Care Hospital Foodservice Patient Satisfaction Questionnaire (ACHFPSQ), which has reportedly been used 
elsewhere in Australia (Fallon et al. 2007) and also in Italy (Messina et al.2013). The questionnaire items were derived from the academic literature, were evaluated against other instruments, and were subjected to statistical treatments such as factor and reliability analysis. The five-factor structure reported by Capra's group is also shown in Table 1.

Table 1 about here please

Other researchers using quantitative methods to assess patients' satisfaction with hospital meals have placed less focus on producing a generalisable questionnaire. Stanga et al. (2003) used a multiple response format with both open and closed questions. Sahin et al. (2006) used a ten-item questionnaire somewhat similar to that of Capra et al. (2005), which asked patients to rate their satisfaction with various aspects of food quality, presentation and service. Hwang et al. (2003) employed a modified SERVQUAL questionnaire, although this was much more concerned with aspects of the food, rather than with the other classical service attributes reliability, responsiveness, assurance and empathy. Johns et al. (2010) used the profile accumulation technique (Johns and LeeRoss, 1996), to assess the relative importance of different aspects of the hospital meal experience. Table 1 shows the relative importance of aspects of the meal experience reported by each of these groups. In all cases the tangible qualities of food were reported as most important, followed variously by the food's appearance and the service. Table 1 compares the findings of these different authors in terms of the relative importance of different factors. The factors themselves of course depend upon the nature and wording of the questions, none of which were derived from prior interviews or focus groups, and probably for this reason, the relative importance of food, service and other factors are quite diverse. Most of these studies claim to reflect the patient's point of view, yet none report fully upon the nature or wording of the questions or the designation of the factors derived from them. observation and interviews of patients in Australia (Walton et al. 2013) the UK (Johns et al. 2013) 
and Iran (Jessri et al. 2011)all offer insights into patients' satisfaction with the meal experience, although these studies were mainly concerned with understanding the impact of the provision of hospital meals upon patients' malnutrition. A similar type of study, but specifically focused on patients' access to food has been conducted in the UK by Naithani et al. (2008). Studies by Watters et al. (2003) and Hartwell et al. (2013) in Canada and the UK respectively focused on the experience of eating in hospital from a more patient-centred point of view, for instance the focus groups set up by Watters et al. listed health, quality, freshness, and appropriateness, variety, selection, and choice, inability to provide feedback, menu errors, accessibility to food, tray layout and waste as concerns about the meal experience. Justesen et al. (2014) used participant-driven-photo-elicitation (participants completed a photo-essay and were interviewed) to examine the hospital meal experience in Denmark. Studies such as these can perhaps truly claim to represent the patient's viewpoint. They provide many valuable insights into what it is like to take meals in hospital, and like the quantitative studies discussed above, they suggest that the most important aspect of the meal experience is the quality of the food. However, they used a small sample of patients and the data produced, though rich, were complex. It is impossible from such studies to identify the relative importance of the factors contributing to patients' satisfaction.

Authors have examined various aspects of food service and consumption in hospital with a view to improving the meal experience. These studies include minutiae of service, such as where food is placed and whether it is unwrapped (Walton et al. 2013), the location of facilities for augmenting meals (Jessri et al. 2011) protection of meal times from interruptions such as medical rounds (Hickson et al. 2011). Edwards \& Hartwell (2004) report the influence of different eating positions on patients' meal experience, and Hartwell \& Edwards (2003) compare the efficiency of different hospital food service systems. Johns et al. (2013) compare food production and service in prisons and hospitals, concluding that the most important factor in both is the timely delivery of food to the recipients, which avoids deterioration of the food. Each of these studies concentrates upon one 
environment). What is not known is the nature of the perceived components of the meal experience and the relevant importance of each to the whole. This knowledge would enable research to be prioritised for maximum effect.

The present study sought to identify and examine all perceived aspects of the meal experience from the patient's viewpoint and to quantify the impact of each one. This was done by interviewing patients to produce a questionnaire which was successively refined. Survey results were subjected to statistical treatments, including factor and cluster analysis to identify contributing factors, and multiple regression to identify the impact of the factors upon satisfaction.

\section{Materials and Method}

The hospital used as a case study had 42 catering staff who prepared the meals for all the wards, providing over 3000 patient meals per day. In addition, they supplied the day wards with cold lunches and snacks and provided meals for two public restaurants used by staff, visitors and some ambulant patients. The hospital used 4 sets of seasonal menus throughout the year on a two-weekly cycle. Under normal ward practice, patients ordered their food 24 hours before the corresponding mealtime by filling in printed forms, and these individual food orders were consolidated by ward staff and telephoned to the kitchen as a bulk order for the following day. Bulk orders were then entered into a computer system for the kitchen to action. Meals prepared in the main hospital kitchen were transported in heated trolleys by porters to the corridors of the individual wards. They were left there for ward staff to bring them onto the wards. Health Care Assistants or Ward Hostesses then served individual patients by their bedside and at this point there might be an opportunity for patients to amend their selection. After meal service was over the trolleys were returned to the corridors and collected by the porters, who returned them to the kitchen. 
A hospital dining experience questionnaire was produced as follows. Preparation and delivery of

101

102 three breakfast, three lunch and three dinner services to patients on two acute orthopaedic wards were observed and extensive notes taken. A purposive sample of 30 patients was chosen for the interview and questionnaire survey using consenting inpatients drawn from a list provided by clinical leaders of the two wards. Those chosen were in the convalescence stage of their recovery, and all met the following criteria. They were over 18 years of age, with no notable physical, cognitive or emotional conditions which might influence their food consumption, and with their appetite unaffected by their medical condition or medication. Their first language was English, they had eaten food on the ward for a minimum of 48 hours previously and they had an anticipated minimum stay of 5 days. Semi-structured interviews conducted at the patients' bedsides aimed to identify the factors influencing patients' enjoyment of their meals, together with issues that patients felt would enhance their mealtime experience in hospital. Additional interviews were conducted with 18 stakeholders, including catering staff, clinical managers and medical staff, ward hostesses, and relevant administrators. Interview transcripts were analysed thematically using NVIVO software, validating issues that arose by reference between samples, regular reviews of the raw data and comparison with the findings of previous research.

A 37 item draft questionnaire was drawn up using these qualitative findings and administered by a researcher to a pilot group of 70 patients attending pre admission clinics. Following this pilot the original 5 point Likert response set was replaced with a 7 point scale, to achieve greater variance and less skewed data (Dawes 2007). In addition, certain questions were reworded or removed, and negatively worded questions were spaced more evenly to reduce respondent confusion (De Vellis 2003). Overall, however, changes were kept to a minimum, partly to avoid the need for further ethical clearance, and partly because the pilot already indicated a high Cronbach's alpha (0.86). A member of the local NHS Research and Development Support Unit who was experienced in the design of questionnaires for the evaluation of clinical services provided support throughout the development of the questionnaire. 
The final draft of the questionnaire consisted of three parts, eliciting:

attitude responses (scaled 1-5) to 17 specific aspects of hospital food and service and to the experience as a whole, and;

8 dining preferences, also scaled 1-5.

It was administered to a purposive sample of 325 orthopaedic ward patients selected as discussed above. The hospital was an Acute Care Hospital with 26 wards including medical, elective surgery, maternity and intensive care. Data were collected from the orthopaedic wards as these patients tended to stay longer and their medical condition would not interfere with food consumption. Thus they were much more likely to match the criteria discussed above than patients on the other wards. For these reasons, orthopaedic patients tended to be more capable of independent judgement, and indeed were often highly critical, as evidenced by past surveys conducted by the catering manager. It was considered that orthopaedic patients would have greater experience of, and would be more able to comment upon the food service system from the point of view of patients as a whole. The wards selected were also the last to receive meals, being at the end of the trolley runs, and therefore the research setting constituted the worst-case food service experience.

Respondents either completed the questionnaire by hand or were helped to do so by a researcher. Some patients were unable to complete the questionnaires, due to being called away for diagnostic tests such as x-rays, but in the end 296 usable questionnaires were received, which were transcribed, and the negatively items reversed in sense. These data were analysed using SPSS

147 (Statistical Package for Social Sciences, version 20, Chicago, IL, USA). The robustness of the questionnaire being assessed using Cronbach's alpha. Individual items were reduced to factors using exploratory factor analysis and the effectiveness of both the items and factors in predicting overall 
satisfaction was assessed using multiple regression. $\mathrm{K}$ means cluster analysis was employed to identify preference patterns among the cases.

152

Results

There were 120 responses from males and 176 from females ( $40.5 \%$ and $59.5 \%$ respectively). The mean age was 69.1, with the minimum 25 and the maximum 94. Respondents who had eaten hospital food on another occasion within the previous year numbered 207 (69.9\%) and 68 (23.0\%) had not eaten it for a year or more. Only two individuals $(0.7 \%)$ said they had never eaten hospital food before.

Cronbach's alpha was 0.835 for the attitude responses, 0.499 for the dining preferences and 0.765 for the attitudes and dining preferences combined. Exploratory factor analysis produced a sevenfactor structure. However, the seventh factor consisted of the single preference item "Eat my meals in bed". Eating in bed has been shown not to influence patients' satisfaction with food service (Edwards \& Hartwell, 2004) and this item was accordingly dropped. When this was done, the Cronbach's alpha value of the remaining preference items rose to 0.555 , and for the preferences and attitudes combined it rose to 0.766 . The resulting six factor structure is shown in Table 2 .

Table 2 about here please

There was cross-loading between factors 1 and 2, on items "Tasty food" and "Meals served at the right temperature" suggesting that respondents associated these qualities with the way the food was served, as well as regarding them as properties of the food itself. That the item "Smells and odours [do not] spoil enjoyment" loaded significantly on factor 6 (staff) suggests that somehow this aspect was associated by patients with staff behaviour, probably through the way the ward operated. Cronbach's alpha values for factors 5 (social) and 6 (staff) were .498 and .409 respectively, and thus below the value (.500) usually regarded as acceptable for factor membership. However, these factors were produced whether the factors were extracted by PCA or least-squares, 
and whether orthogonal or oblique rotation was employed, and they were therefore considered robust enough to be retained in the analysis. The six factors were examined for demographic differences using t-test and one-way ANOVA. Males scored higher than females on overall experience and on all factors except situation, but the difference was only significant $(p<0.05)$ on food and ward. There were no significant differences (one way ANOVA; $p<0.05$ ) among the five age groups on overall experience or on any of the factors, although older individuals tended to have a more positive view. The five "previous experience" groups showed significant differences (one way ANOVA; $p<0.05$ ) on factors food and situation. These five groups were amalgamated to give two roughly equal sized groups having greater and lesser experience of hospital meals, and these showed differences (independent samples t-test; $p<0.05$ ) on overall experience, and service, as well as on food and situation. The less experienced respondents tended to be more positive on overall experience, food and service. On social, staff and ward, the more experienced respondents tended to be more positive.

In order to assess their respective influence, the factors were regressed against patients' overall experience of meals at the hospital, producing the coefficients shown in Table 3. (Cluster membership, which is included in this table, is discussed below.)

Table 3 about here please

In order to identify differences between the preferences and needs of groups of individuals, the cases were clustered on the basis of the six factors using the k-means method. Five solutions with between two to six clusters were explored. The three-cluster system showed greater discrimination than the two-cluster, but those with four or more clusters had more than four overlaps between factors and were therefore rejected. Factor means for the three-cluster system are shown in Figure 1. 
Cluster $1(\mathrm{~N}=62)$ was characterised by higher mean scores than Cluster 2 on overall experience, and on all factors except situation. Cluster $2(\mathrm{~N}=41)$ had relatively low mean scores for overall experience and for all factors. Cluster $3(\mathrm{~N}=107)$ had high values comparable to Cluster 1 for all means apart from situation, where it scored very low. There was a significant preponderance of males in Clusters 1 and 3 (Chi square $p=0.0003$ ), but there were no significant trends among the clusters in terms of age or previous experience of hospital meals.

Since no other relationship could be determined, it was assumed that the means in Figure 1 represented different individual requirements or preferences. Cluster membership was therefore replaced by the mean of the factors contributing to each cluster, and this permitted the cluster outcome to be included in the multiple regression, as shown in Table 3, where it appears half-way down the list in terms of beta value.

Discussion

Preparation of the questionnaire used in this study took the fullest possible account of the patients' point of view. It involved observations and interviews with patients and it was refined using a patient centred pilot study. The demographic characteristics of the sample suggest that it was representative of the population. The robustness of the factor structure obtained from this instrument may be questioned in terms of reliability measures such as Cronbach's alpha (values only .498 and .409 for factors 5 and 6 respectively), but was supported by the persistence of the factors and their intuitive nature. At face value, the regression results suggest that the factors contributing to the hospital meal experience may be represented by a model such as that shown in Figure 2 .

This model emphasises food quality and service quality as the main contributors to the experience, listing the others in the order of their regression $\beta$ values. However, the $t$ and $p$ values (see Table 3 ) indicate that for all factors with $\beta$ values less than 0.205 (i.e. below that of service) the proportion of 
variance explained by the regression, and hence the certainty of their placing was below $5 \%$ statistical significance. Therefore the order shown in Figure 3 for items above service is speculative at best. Nevertheless it represents all the relevant factors and at the same time agrees with the findings of other authors (see Table 1), to the extent that it lists food quality (or its elements) as the most important contributor, followed by service (or its elements).

The notion of service in hospital dining is clearly complex. In the present study, food tastiness and temperature loaded highly on the factor Food, but also had relatively high loadings on Service (see Table 2). Other authors have reported both tangible (timeliness, reliability, crockery) and interpersonal aspects as "service" (Dubé et al., 1994; Stanga et al., 2003; Sahin et al., 2006; Hwang et al., 2003). Johns et al. (2010) found that patients resented the constraints of ordering and eating at specific times. They were sensitive to delays caused by slow service but grateful of opportunities to speak with non-medical food service staff and acutely aware that food service sometimes placed further stresses on already stretched nursing staff. Johns et al. (2013) report the importance of timely service in ensuring that food arrives at the patient's bedside in a palatable condition. Although the present study used the word service, it was probably as ill-fitted to patients' actual experience in this study as in research published by other authors. However, it is difficult to see how this term may be avoided, given its ubiquity in consumer-related discourse. Possibly a different approach, such as participant-driven-photo-elicitation (Justesen et al., 2014) might shed a clearer light on the nature of service in the hospital environment.

Individual characteristics of patients likely to influence satisfaction with food service include their gender, their personal preferences, specific diseases and medication (Cardello et al., 1996), their ethnic backgrounds (Jessri et al., 2011), their physical state (which may affect their perception of the food, or their ability to feed themselves) (Corish \& Kennedy, 2000) and their age (Stephen et al. 1997; Johns et al. 2010). The present study sought an empirical grouping that might encompass at least some of these individual aspects, and this was eventually identified as the three-cluster system 
shown in Figure 1, based upon the measured attitudes and preferences. The clusters differentiated between genders, but not between age or experience groups, even though these groupings were shown to influence the factors. It might be possible to identify a more robust measure of individual preference, as a stronger predictor of overall satisfaction. However, to date this has been the only study to attempt such a measurement.

Conclusion

This was the first study to attempt evaluation of patients' satisfaction with hospital food service on a holistic and patient-focused basis. It demonstrated from first principles that food quality, followed by service quality were the most important predictors of customer satisfaction, thereby confirming findings of some previous authors. After this, the social environment, the personal characteristics of the patient and the immediate eating environment were the most important factors. However only food and service contributed sufficiently to the total variance to produce a statistically significant relationship, so that the order of the latter factors cannot be guaranteed. Nevertheless, from a practical point of view, the results suggest that improving the quality of the food and the timeliness with which it is provided remain the most important objectives of hospital food service. They therefore emphasise the significance of efficient production and transport of hospital food highlighted by other authors (Walton, et al., 2013; Johns et al., 2013; Jessri et al., 2011) A major problem with this kind of quantification is ambiguity of questionnaire items. The present study sought rigorously to avoid this by deriving and purifying the questions with the direct help of patients in the hospital. The fact that this was not completely successful suggests that words such as "service" are used in multiple senses, even when referring to a single recognisable issue such as the provision of food to hospital patients. It is possible that a new vocabulary needs to be developed, and to become commonly accepted by patients, before this particular line of research can be moved forward. Nevertheless there must be approaches which can be used profitably in the study of the hospital meal experience. Two such that have already been tested are profile accumulation 
technique (Johns et al., 2010) and participant-driven-photo-elicitation (Justesen et al., 2014), discussed above. It seems likely that, augmented with observation and interview techniques, these may make it possible to establish the relative importance of the different factors.

Notwithstanding the ambiguity of terms and the consequent lack of definition of some of the terms identified here as contributing to patients' experience of meals in hospital, the priorities for food service practice are clear. The relationship between the timeliness of food production and service and the quality of food reaching the patients has been pointed out by various authors (Johns et al., 2014; Jessri et al. 2011; Hartwell \& Edwards, 2003). This article has further highlighted the urgent need for hospital service managers to address the factors that continue to impede the rapid transfer of food from kitchens to wards, to the detriment of its quality.

\section{References}

Andersson, A-C., Elg, M., Perseius, K-I. \& Idvall, E. (2013). Evaluating a questionnaire to measure improvement initiatives in Swedish healthcare. BMC Health Services Research, 13 (1), 1-11. BAPEN (2007). Nutrition Screening Survey in the UK in 2007: Nutrition Screening Survey and Audit of Adults on Admission to Hospitals, Care Homes and Mental Health Units. Available from: http://www.bapen.org.uk/pdfs/nsw/nsw_report2008-09.pdf [Accessed 28 August 2008].

Bélanger M-C. \& Dubé L. (1996). The emotional experience of hospitalization: its moderators and its role in patient satisfaction with foodservices. J Am Diet Assoc, 96, 354-60.

Capra, S., Wright, O., Sardie, M., Bauer, J. \& Askew, D. (2005). The acute hospital foodservice patient satisfaction questionnaire: the development of a valid and reliable tool to measure patient satisfaction with acute care hospital foodservices. Foodservice Research International,16 (1), 1-14. 
294 Cardello, A. V., Bell, R. \& Kramer, M. (1996). Attitudes of consumers toward military and other

295 institutional foods. Food Quality and Preference, 7, 7-20.

296 Corish, C. A. \& Kennedy, N. P. (2000). Protein-energy undernutrition in hospital in-patients. British

297 Journal of Nutrition, 83(6), 575-591.

298 Dawes, J. (2007). Do data characteristics change according to the number of scale points used?

299 International Journal of Market Research, 50, 61-77.

300 De Vellis, R.F. (2003). Scale development: theory and applications. 2nd ed. London: Sage.

301 Dubé L, Trudeau E \& Bélanger M-C. (1994). Determining the complexity of patient satisfaction with 302 foodservices. J Am Diet Assoc, 94, 394-400.

303 Edwards \& Hartwell (2004). A comparison of energy intake between eating positions in a NHS

304 hospital - a pilot study. Appetite, 43, 323-325.

305 European Nutrition for Health Alliance (2008). European Nutrition for Health Alliance launches

306 campaign to highlight the challenge of Malnutrition in society. Available from:

307 http://www.european-nutrition.org [Accessed 4 November 2008].

308 Fallon, A., Gurr, S., Hannan-Jones, M. \& Bauer, J. D. (2007). Use of the acute care hospital

309 foodservice patient satisfaction questionnaire to monitor trends in patient satisfaction with

310 foodservice at an acute care private hospital. Nutrition \& Dietetics, 65, 41-45.

312 Feldblum, I., German, L., Bilenko, N., Shahar, A., Enten, R., Greenberg, D., Harman, I., Castel, H. \&

313 Shahar, D. R. (2009). Applied nutritional investigation: Nutritional risk and health care use before and

314 after an acute hospitalization among the elderly. Nutrition, 25(4), 415-420. 
Hartwell, H. \& Edwards, J.S.A (2003) comparative analysis of 'plated' and 'bulk trolley' hospital food service systems. Food Service Technology. Vol. 3 Issue 3/4, p133-142.

Hartwell, H. J., Shepherd, P. A. \& Edwards, J.S.A. (2013). Effects of a hospital ward eating environment on patients' mealtime experience: A pilot study. Nutrition \& Dietetics, 70(4), 332.

Hickson, M., Connolly, A. \& Whelan, K. (2011). Impact of protected mealtimes on ward mealtime environment, patient experience and nutrient intake in hospitalised patients. Journal of Human Nutrition and Dietetics, 24(4), 370-374.

Hwang, L-J. J., Eves, A. \& Desombre, T. (2003). Gap analysis of patient mealservice perceptions. International Journal of Health Care Quality Assurance, 16(3), 143-53. difference: patients' views of hospital food service in Iran. Appetite, 57 (2), 530-533. comparative analysis of food service systems. Appetite, 68, 45-50. patients' viewpoint. Appetite, 54 (1), 181-185. Johns, N. \& Lee-Ross, D. (1996). Profile accumulation: a quality assessment technique for hospitality SMEs. in Teare R. \& Armistead, C (eds.). Services Management: New Directions and Perspectives, London: Cassell, 101-104. Justesen, L., Mikkelsen, B. E. \& Gyimóthy, S. (2014). Understanding hospital meal experiences by means of participant-driven-photo-elicitation. Appetite, 75, 30-39 

experiences of access to food: a qualitative interview and observational study. Health Expectations, 11(3), 294-303.

341 Russell, N. C., Wallace, L. M. \& Ketley, D. (2011). Evaluation and measurement

342 for improvement in service-level quality improvement initiatives. Health Services Management 343 Research, 24(4), 182-9.

344 Sahin, B., Demir, C., Celik, Y. \& Teke, A. K. (2006). Factors affecting satisfaction level with the food 345 services in a military hospital. J. Med. Syst. 30, 381-387.

347 European healthcare systems. Quality \& Safety In Health Care, 18 (Suppl 1), 122-7. survey of patients' perceptions. Clinical Nutrition, 22(3), 241-246. wastage and intake in elderly hospital patients. Proceedings of the Nutrition Society, 56, $220 \mathrm{~A}$. 

satisfaction. Australian Health Review, 26(1): 70-75.

361

362 